\title{
HER2-Targeted PET Imaging and Therapy of Hyaluronan-Masked HER2-Overexpressing Breast Cancer
}

\author{
Patricia M. R. Pereira ${ }^{\dagger}$, Ashwin Ragupathi ${ }^{\dagger}$, Shayla Shmuel ${ }^{\dagger}$, Komal Mandleywala $^{\dagger}$, Nerissa \\ T. Viola ${ }^{*}, \neq$, Jason S. Lewis ${ }^{\star}, \dagger, \S, \|, \perp, \#$ \\ tDepartment of Radiology, Memorial Sloan Kettering Cancer Center, New York, New York 10065, \\ United States \\ ‡Department of Oncology, Karmanos Cancer Institute, Wayne State University, 4100 John R \\ Street, Detroit, Michigan 48201, United States \\ $\S$ Molecular Pharmacology Program, Memorial Sloan Kettering Cancer Center, New York, New \\ York, United States \\ "Department of Pharmacology, Weill Cornell Medical College, New York, New York, United States \\ ${ }^{\perp}$ Department of Radiology, Weill Cornell Medical College, New York, New York, United States \\ \#Radiochemistry and Molecular Imaging Probes Core, Memorial Sloan Kettering Cancer Center, \\ New York, New York, United States
}

\begin{abstract}
Human epidermal growth factor receptor 2 (HER2) is a biomarker in breast cancer, and its overexpression is required to initiate therapies using HER2-targeted antibodies. Although trastuzumab is one of the most effective therapeutic antibodies in HER2-overexpressing breast cancer, a significant number of patients do not benefit from this therapy due to inherent or acquired resistance mechanisms. One reported mechanism of resistance is the steric hindering effect caused by the polymeric complex formed between hyaluronan and CD44, thus preventing trastuzumab from binding to HER2. Hyaluronan/CD44 contributes as an obstacle for trastuzumab to bind HER2, but it is also involved in HER2 internalization. In this study, we used zirconium-89 $\left({ }^{89} \mathrm{Zr}\right.$ )-labeled trastuzumab immunoPET to investigate whether degradation of hyaluronan can resensitize HER2-overexpressing breast cancer cells to trastuzumab. Targeted degradation of endogenously produced hyaluronan and inhibition of its synthesis were achieved by treating trastuzumab-resistant JIMT1 breast cancer cells with hyaluronidase (HLX) and 4methylumbelliferone (4MU). The 4MU/HLX treatment reduced HER2 internalization by depleting hyaluronan/CD44 and the caveolin-1 (CAV1) endocytic protein, resulting in enhanced membrane-
\end{abstract}

\footnotetext{
*Corresponding Authors: violan@karmanos.org. Phone: +1 313-576-8309. Fax: +1 313-576-8928. lewisj2@mskcc.org. Phone: +1 646-888-3038. Fax: 646-888-3059.

Supporting Information

The Supporting Information is available free of charge at https://pubs.acs.org/doi/10.1021/acs.molpharmaceut.9b01091.

Tumor volumes in control and 4MU/HLX-treated tumors during the 3 weeks of experimental procedures of imaging studies; full biodistribution and autoradiography of $\left[{ }^{89} \mathrm{Zr}\right] \mathrm{Zr}$-DFO-trastuzumab in control and 4MU/HLX-treated HER2-expressing JIMT1 tumor xenografts; ${ }^{89} \mathrm{Zr}$-labeled IgG tumor uptake in control and 4MU/HLX treated JIMT1 tumors; Kaplan-Meier survival curves of mice bearing JIMT1 breast tumors and treated with saline, 4MU, HLX, or 4MU/HLX (PDF)

The authors declare no competing financial interest.
} 
bound ${ }^{89} \mathrm{Zr}$-labeled trastuzumab. 4MU/HLX enhanced trastuzumab tumor uptake, as evidenced by increased tumor binding of the ${ }^{89} \mathrm{Zr}$-labeled trastuzumab in JIMT1 tumor xenografts. In vitro mechanistic studies demonstrated a decrease in HER2-mediated oncogenic signaling upon cell treatment with 4MU/HLX. Importantly, 4MU/HLX enhanced trastuzumab efficacy in JIMT1 xenografts. These data showed the utility of ${ }^{89} \mathrm{Zr}$-labeled trastuzumab as a PET imaging agent to monitor the affinity of the antibody to HER2 during CD44/hyaluronan-specific inhibition with the overall goal of improving trastuzumab therapy.

\section{Graphical Abstract}

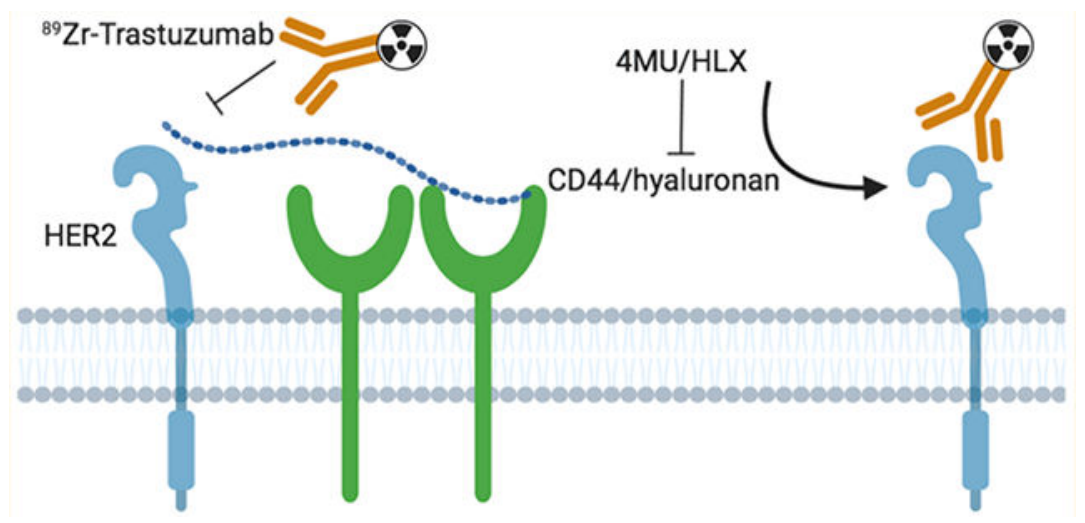

\section{Keywords}

HER2; CD44; hyaluronan; CAV1; trastuzumab; PET imaging

\section{INTRODUCTION}

Human epidermal growth factor receptor 2 (HER2) is a $185 \mathrm{kDa}$ receptor tyrosine kinase (RTK) that belongs to a family consisting of three other RTK members (EGFR or HER1, HER3, and HER4). ${ }^{1,2}$ This RTK family is known as the human epidermal growth factor receptors or HERs. The binding of distinct peptide ligands to the extracellular binding domains of EGFR, HER3, and HER4 induces receptor homo- and heterodimerization. ${ }^{3-5}$ Contrary to the other members of the HER family, HER2 appears not to have an established binding ligand, but it can dimerize with other members of the HER family. HER2 dimerization induces downstream oncogenic signaling, which results in cell proliferation, migration, invasion, survival, and resistance to apoptosis. ${ }^{6}$

The gene ERBB2 (also known as neu) encoding the HER2 protein is discovered to be amplified in $15-20 \%$ of newly diagnosed breast cancers. ${ }^{7}$ In women with breast cancer, $E R B B 2$ gene amplification and HER2 protein overexpression associates with a worse prognosis. ${ }^{8}$ HER 2 is not only a tumor biomarker in patients with breast cancer but also a therapeutic target. Several therapeutic antibodies targeting HER2 have been developed and clinically translated for patients with HER2-overexpressing breast cancer. ${ }^{2}$ The murine monoclonal antibody 4D5, developed in 1988, against the extracellular domain of HER2, demonstrated significant efficacy in HER2-expressing breast cancer cells. ${ }^{9}$ The 4D5 
antibody was further "humanized", resulting in the generation of trastuzumab (Herceptin), a humanized IgG1 $\kappa$-light chain monoclonal antibody. ${ }^{10}$ Trastuzumab increases overall survival in patients with HER2-positive metastatic breast cancers and reduces mortality when administrated in the adjuvant setting. ${ }^{11}$ In the context of oncologic molecular imaging, trastuzumab radiolabeled with zirconium- 89 ( ${ }^{89} \mathrm{Zr}$-labeled trastuzumab) is an effective agent for selecting patients for HER2-targeted therapy and therapeutic monitoring response. ${ }^{12-16}$

Inherent and acquired tumor resistance occurs during treatment with trastuzumab, and several mechanisms contribute to this resistance: (i) upregulation of HER2-mediated downstream oncogenic signaling, ${ }^{17}$ (ii) RTK coactivation upon treatment, ${ }^{15}$ (iii) obstacles preventing trastuzumab to bind HER2, ${ }^{18-21}$ and (iv) failure to induce an antibody-dependent cell-mediated cytotoxicity (ADCC) mechanism. ${ }^{17}$ In the context of obstacles blocking the binding between trastuzumab and HER2, the CD44/hyaluronan polymer complex hinders the access of trastuzumab to HER2. ${ }^{17,18,22}$ Hyaluronan is a glycosaminoglycan that creates a volume barrier within the extracellular matrix. CD44 is a transmembrane protein and the main hyaluronan receptor. ${ }^{23}$ Binding of hyaluronan to CD44 and further interaction between CD44, Grb2, Vav2, and HER2 activates CD44-mediated downstream signaling including the PI3K-AKT survival pathway. ${ }^{24}$ Previous studies demonstrated that the combination of trastuzumab with the inhibitor of hyaluronan synthesis, 4-methylumbelliferone (4MU), improves antibody efficacy in HER2-expressing JIMT1 breast tumor xenografts. ${ }^{18}$ Endogeneous hyaluronan can be degraded by hyaluronidase (HLX) in a local, reversible, and transient manner to increase the diffusion of locally injected drugs. ${ }^{25,26}$ In the context of HLX to enhance antibody accumulation in tumors, different HLX/trastuzumab approaches have been investigated, ${ }^{27,28}$ and the hyaluronidase-oysk (Herceptin Hylecta, a combination of trastuzumab with HLX) was recently FDA approved in the treatment of $\mathrm{HER}_{2}{ }^{+}$breast cancer.

In this study, we used HER2-targeted molecular imaging to annotate the effects of the 4MU/ HLX-mediated depletion of the CD44/hyaluronan complex on trastuzumab binding and resensitization of HER2-overexpressing resistant JIMT1 breast cancer xenografts.

\section{EXPERIMENTAL SECTION}

\section{Cell Lines and Treatments.}

JIMT1 breast cancer cells were obtained from the Leibniz Institute DSMZ-German Collection of Microorganisms and Cell Cultures ACC 589. JIMT1 cells were used within 15 passages, confirmed to be mycoplasma free, and authenticated by the Memorial SloanKettering Cancer Center (MSK) Integrated Genomics Operation Core using short tandem repeat analysis. Cells were maintained at $37{ }^{\circ} \mathrm{C}$ in a humidified atmosphere at $5 \% \mathrm{CO}_{2}$ in Dulbecco's modified Eagle medium: Nutrient Mixture F-12 (DMEM/F-12) supplemented with $10 \%$ fetal calf serum (FCS), $2 \mathrm{mM}$ L-glutamine, $0.01 \mathrm{~g} / \mathrm{L}$ insulin, and $100 \mathrm{units} / \mathrm{mL}$ of both penicillin and streptomycin.

JIMT1 cells were incubated with $1 \mathrm{mM} 4 \mathrm{MU}$ (Sigma, M1381) for $48 \mathrm{~h}$ prior to the addition of trastuzumab. For the experiments with HLX (Calbiochem, HX0514), cells were incubated with 1 Unit HLX for $2 \mathrm{~h}$ in the presence of trastuzumab. Control experiments were 
performed by incubating JIMT1 cancer cells in the medium before the addition of trastuzumab.

\section{Western Blot Analysis.}

Whole-protein extracts from control or treated JIMT1 cells were obtained as previously described by cell scraping at $4{ }^{\circ} \mathrm{C}$ in a radioimmunoprecipitation assay buffer. ${ }^{20}$ Following electrophoresis and transfer to nitrocellulose membranes (Thermo Fisher Scientific, IB23001), the blots were incubated in 5\% w/v bovine serum albumin (BSA, Sigma, A7030) in Tris-buffered saline buffer-Tween (TBS-T, Cell Signaling Technology, 9997S) and probed with mouse anti- $\beta$-actin 1:20 000 (Sigma, A1978), rabbit anti-HER2 1:800 (Abcam, ab131490), rabbit anti-pHER2 1:800 (Abcam, ab53290), rabbit anti-EGFR 1:1000 (Abcam, ab52894), rabbit anti-pEGFR 1:500 (Abcam, ab40815), rabbit anti-HER3 1:500 (Abcam, ab32121), rabbit anti-pHER3 1:2500 (Abcam, ab76469), rabbit anti-AKT 1:1000 (Cell Signaling, 9272), rabbit anti-pAKT 1:2000 (Cell Signaling, 4060), mouse anti-ERK 1:1000 (Thermo Fisher Scientific, 14-9108-82), rabbit anti-pERK 1:500 (Thermo Fisher Scientific, 700012), rabbit anti-CD44 1:1000 (Abcam, ab189524), and rabbit anti-CAV1 1:500 (Abcam, ab2910) antibodies. After the antibodies were incubated and washed, the membranes were incubated with IRDye800CW anti-Rabbit (925-32211) or anti-Mouse (925-32210) IgG 1:15 000 (LI-COR Biosciences) and imaged on the Odyssey Infrared Imaging System (LI-COR Biosciences) followed by densitometric analysis using Fiji software (https://imagej.net/Fiji).

\section{Immunoprecipitation Assays.}

Total cellular protein (500 $\mu \mathrm{L}$ of RIPA buffer containing $200 \mu \mathrm{g}$ of protein) was incubated with $10 \mu \mathrm{g}$ of primary antibody Neu (F-11) agarose conjugate (Santa Cruz Biotechnology, sc-7301 AC) overnight at $4{ }^{\circ} \mathrm{C}$ with gentle rotation. The pellet (containing the immunoprecipitated fraction) was collected by centrifugation at $1000 \mathrm{~g}$ for $30 \mathrm{~s}$ at $4{ }^{\circ} \mathrm{C}$ and washed three times with RIPA buffer before resuspension in Laemmli buffer.

\section{Immunofluorescence Microscopy.}

JIMT1 tumors were collected and preserved in the OCT matrix (Tissue-Tek Optimal Cutting Temperature Compound, Fisher Scientific, 23-730-571), gently frozen in liquid nitrogen, and stored at $-80{ }^{\circ} \mathrm{C}$ for $24 \mathrm{~h}$. Samples were then left for $30 \mathrm{~min}$ at $-20^{\circ} \mathrm{C}$ and processed into $10 \mu \mathrm{m}$ thick sections using a microtome at $-20^{\circ} \mathrm{C}$. Sections were air-dried and fixed in $4 \%$ paraformaldehyde (PFA) for $15 \mathrm{~min}$. The sections were then washed with phosphatebuffered saline (PBS), permeabilized for 30 min with $0.25 \% \mathrm{v} / \mathrm{v}$ Triton X-100 (Tx-100, Sigma, T8787) in PBS with $0.02 \%$ w/v BSA (PBS/BSA), and blocked with $10 \% \mathrm{v} / \mathrm{v}$ normal goat serum (Thermo Fischer Scientific, 50062Z) before incubation overnight at $4{ }^{\circ} \mathrm{C}$ with primary antibodies $(1: 100 \mathrm{v} / \mathrm{v})$ rabbit anti-HER2, rabbit anti-CAV1, or rabbit anti-CD44. Sections were then rinsed with PBS and incubated with DAPI (1:1000 v/v, Sigma, D9542) for nuclear staining and the secondary antirabbit fluorescent antibodies (1:300 v/v, Goat anti-Rabbit IgG H+L Cross-Adsorbed Secondary Antibody, Alexa Fluor 488, A-11008) for $1 \mathrm{~h}$ at room temperature. The coverslips were mounted using the glycergel mounting medium (Agilent Technologies, C056330) and sealed with nail polish. The samples were stored at $4{ }^{\circ} \mathrm{C}$ until the acquisition of images by fluorescence microscopy. 


\section{Trastuzumab DFO Conjugation and Radiolabeling.}

Trastuzumab was conjugated with the chelate $p$-isothiocyanatobenzyl-desferrioxamine (DFO-Bz-NCS; Macrocyclics, B-705) and then radiolabeled with zirconium-89 $\left({ }^{89} \mathrm{Zr}\right){ }^{29}$

Zirconium- 89 was produced via proton beam bombardment of yttrium foil and isolated with high purity as $\left[{ }^{89} \mathrm{Zr}\right] \mathrm{Zr}$-oxalate at MSKCC..$^{30}\left[{ }^{89} \mathrm{Zr}\right] \mathrm{Zr}$-DFO-trastuzumab with a radiochemical purity (RCP) of $\geq 95 \%$ as determined by instant thin-layer chromatography was used for in vitro and in vivo studies.

\section{Internalization and Binding Assays.}

Control, 4MU, HLX, or 4MU/HLX-treated cells were incubated with a cell culture medium in the presence of $1 \mu \mathrm{M}\left[{ }^{89} \mathrm{Zr}\right] \mathrm{Zr}$-DFO-trastuzumab for $90 \mathrm{~min}$ at $37{ }^{\circ} \mathrm{C}$. The medium containing the noncell-bound radiotracer was removed, and the cells were washed twice with PBS. The cell surface-bound radiotracer was collected by incubation at $4{ }^{\circ} \mathrm{C}$ for $5 \mathrm{~min}$ in 0.2 $\mathrm{M}$ glycine buffer containing $0.15 \mathrm{M}$ sodium chloride $(\mathrm{NaCl})$ and $4 \mathrm{M}$ urea at $\mathrm{pH} 2.5$. The internalized fraction was obtained at room temperature after cell lysis with $1 \mathrm{M}$ sodium hydroxide $(\mathrm{NaOH})$. The three collected fractions were measured for radioactivity on a gamma counter calibrated for ${ }^{89} \mathrm{Zr}$.

For the binding assays, control, 4MU, HLX, or 4MU/HLX-treated cells were incubated with ${ }^{89} \mathrm{Zr}$-labeled trastuzumab $(0-256 \mathrm{nM})$ in PBS ( $\left.\mathrm{pH} 7.5\right)$ containing $1 \%$ w/v human serum albumin (Sigma) and $0.1 \% \mathrm{w} / \mathrm{v}$ sodium azide (Acros Organics) for $2 \mathrm{~h}$ at $4{ }^{\circ} \mathrm{C}$. Unbound radioactivity was removed, and cells were washed three times with PBS. The cells were solubilized in $100 \mathrm{mM} \mathrm{NaOH}$, and the total cell-bound radioactivity was measured on a $\gamma$ counter calibrated for ${ }^{89} \mathrm{Zr}$. Total binding was plotted vs the concentration of ${ }^{89} \mathrm{Zrtrastuzumab}$; the data were fit via nonlinear regression with a one-site binding model in GraphPad Prism 7.00 (www.graphpad.com) to determine $B_{\max }$.

\section{Tumor Xenografts.}

Experiments with animals were conducted according to the guidelines approved by the Research Animal Resource Center and Institutional Animal Care and Use Committee at MSK. The first author (Pereira) has a Category $\mathrm{C}$ accreditation for animal research from the Federation of European Laboratory Animal Science (FELASA). We adhere to the Animal Research: reporting of in vivo experiments (ARRIVE) guidelines and to the guidelines for the welfare and use of animals in cancer research. Female $n u / n u$ mice (8-10 weeks old) were received from Charles River Laboratories and allowed to acclimate to the facility's laboratory conditions for 1 week prior to experimentation. The mice were housed in type II polycarbonate cages, fed a sterilized standard laboratory diet, and received sterile water ad libitum. The animals were housed at approximately $22{ }^{\circ} \mathrm{C}$ and $60 \%$ relative humidity, and a $12 \mathrm{~h}$ light and $12 \mathrm{~h}$ dark cycle was maintained.

The mice were injected subcutaneously on the right shoulder with 5 million JIMT1 cells in a $150 \mu \mathrm{L}$ cell suspension of a 1:1 $\mathrm{v} / \mathrm{v}$ mixture of medium with reconstituted basement membrane (BD Matrigel, BD Biosciences). The tumor volume $\left(V, \mathrm{~mm}^{3}\right)$ was estimated by external vernier caliper measurements of the longest axis, $a(\mathrm{~mm})$, and the axis 
perpendicular to the longest axis, $b(\mathrm{~mm})$. The tumors were assumed to be spheroidal, and the volume was calculated using the equation $V=(4 \pi / 3) \times(a / 2)^{2} \times(b / 2)$.

\section{PET Imaging, Biodistribution, and Autoradiography Studies.}

When the volume of xenografts reached approximately $100 \mathrm{~mm}^{3}$, mice were randomized into groups, and treatments were initiated ( $n=5$ mice per group for biodistribution and $n=3$ mice per group for PET imaging). 4MU was orally administrated $(0.8 \mathrm{mg} / \mathrm{g}$ of mice prepared in $1 \% \mathrm{w} / \mathrm{v}$ arabic gum) every day for 3 weeks. HLX was subcutaneously administered (10 Units in $50 \mu \mathrm{L}$ of PBS) every day in the third week. $\left[{ }^{89} \mathrm{Zr}\right] \mathrm{Zr}$-DFOtrastuzumab (8.14-10.18 MBq, 80-100 $\mu$ g protein) was administered by tail vein injection on day 3 of the third week.

Imaging experiments were conducted on a microPET Focus 120 scanner (Concorde Microsystems). Mice were anesthetized by inhalation of 1.5-2\% isoflurane (Baxter Healthcare) in an oxygen gas mixture 10 min before recording PET images. PET data for each group $(n=3)$ was recorded, with mice under isoflurane anesthesia $(1.5-2 \%)$, in list mode at $48 \mathrm{~h}$ after intravenous injection of $\left[{ }^{89} \mathrm{Zr}\right] \mathrm{Zr}$-DFO-trastuzumab. This imaging time point was previously established by our group as the optimal imaging time post-tracer injection. ${ }^{16,29}$ List mode emission data were sorted into two-dimensional (2D) sinograms via Fourier rebinning; data were normalized to correct for the nonuniform detector response, dead time count losses, and positron branching ratio, but no attenuation, scatter, or partialvolume averaging corrections were applied. A $2 \mathrm{D}$ ordered subset expectation maximization algorithm (OSEM2D; 4 iterations) was used for reconstruction, and each reconstructed image was smoothed by convolution with a $1.5 \mathrm{~mm}$ full width at half-maximum (fwhm) Gaussian filter kernel. All images were visualized in AMIDE 1.0.4 software (http:// amide.sourceforge.net).

Acute biodistribution and autoradiography studies were carried out at $48 \mathrm{~h}$ after intravenous injection of [ $\left.{ }^{89} \mathrm{Zr}\right] \mathrm{Zr}-\mathrm{DFO}$-trastuzumab according to previously reported methods. ${ }^{20,31}$

\section{In Vivo Therapeutic Studies.}

When tumor volumes reached 100 to $300 \mathrm{~mm}^{3}$, mice were randomly grouped into treatment cohorts ( $n=10$ mice per group): control, 4MU, HLX, 4MU/HLX, trastuzumab, trastuzumab/4MU, trastuzumab/HLX, and trastuzumab/4MU/HLX. 4MU was orally administered $(0.8 \mathrm{mg} / \mathrm{g}$ of mice prepared in $1 \% \mathrm{w} / \mathrm{v}$ arabic gum $)$ every day for 5 weeks. 4MU administration started on day 0. HLX was subcutaneously administered (10 Units in $50 \mu \mathrm{L}$ of PBS) every day in the third, fourth, and fifth weeks. Intraperitoneal trastuzumab administration $5 \mathrm{mg} / \mathrm{kg}$ weekly (during 3 weeks) was started on the third week. Tumor volumes were determined twice a week.

\section{Statistical Analysis.}

Data are expressed as mean \pm standard error of the mean (SEM). Groups were compared using the Student's $t$-test. 


\section{RESULTS}

\section{Depletion of Hyaluronan Improves Trastuzumab Binding to JIMT1 Breast Cancer Cells.}

Given that the CD44/hyaluronan polymer blocks trastuzumab binding to the HER2 receptor by masking the HER2 epitope to which trastuzumab binds to, ${ }^{17,18}$ we sought to determine if depletion of CD44/hyaluronan would result in improved binding of trastuzumab to HER2expressing JIMT1 breast cancer cells. JIMT1 breast cancer cells have ERBB2 oncogene amplification, overexpress HER2 mRNA and protein, and are resistant to anti-HER2 therapeutic antibodies. ${ }^{32-35}$ Previous studies have demonstrated a role for the CD44/ hyaluronan complex in the resistance of JIMT1 cells to trastuzumab therapy. ${ }^{18,36}$ In our studies, targeted degradation of endogenous hyaluronan and inhibition of its synthesis were achieved using HLX and 4MU. ${ }^{18,22}$ Cellular fractionation of HLX-treated JIMT1 cells incubated with ${ }^{89} \mathrm{Zr}$-labeled trastuzumab revealed a significant increase $(P<0.001$, Student's $t$-test) in internalized-associated radioactivity (Figure 1A). On the other hand, an increase in membrane-associated ${ }^{89} \mathrm{Zr}$-labeled trastuzumab was observed upon cell treatment with 4MU or 4MU/HLX ( $P<0.001$, Student's $t$-test, Figure 1A). Additional radioligand binding assays confirmed that hyaluronan depletion with 4MU or 4MU/HLX increased trastuzumab binding to JIMT1 breast cancer cells $\left(B_{\max }, \mathrm{P}<0.05\right.$, Student's $t$-test, Figure 1B).

Altogether, the internalization and binding in vitro studies indicate that hyaluronan depletion improves trastuzumab binding on HER2-expressing JIMT1 breast cancer cells.

\section{Depletion of Hyaluronan Increases JIMT1 Tumor Avidity for Trastuzumab.}

Premised on our in vitro findings (Figure 1), we expected that hyaluronan depletion with a combination treatment of 4MU/HLX might result in increased binding of trastuzumab in JIMT1 breast tumor xenografts. To achieve complete in vivo hyaluronan degradation, we used a combination of 4MU/HLX. 4MU inhibits hyaluronan synthesis, and HLX degrades endogenous hyaluronan. In our in vivo studies, we used HER2-targeted PET imaging to monitor the affinity of trastuzumab to HER2-expressing JIMT1 tumors during hyaluronan depletion using a 4MU/HLX combination treatment. Once the tumors reached a volume of $100 \mathrm{~mm}^{3}$, mice were administrated with a combination of 4MU and HLX before the administration of ${ }^{89} \mathrm{Zr}$-labeled trastuzumab (Figure $2 \mathrm{~A}$ ). Since $4 \mathrm{MU}$ is used as a dietary supplement, ${ }^{37}$ our studies were performed using oral administration of $4 \mathrm{MU}(0.8 \mathrm{mg} / \mathrm{g} / \mathrm{day}$, Figure 2A). Based on the FDA's formula of mouse-to-human dose conversion, a 0.8 $\mathrm{mg} / \mathrm{g} /$ day $4 \mathrm{MU}$ dose in mice correspond to human equivalent doses of $3.9 \mathrm{~g} /$ day; these are doses at which $4 \mathrm{MU}$ is consumed by humans for improving liver health. ${ }^{37}$ Previous studies have demonstrated that $4 \mathrm{MU}$ at daily doses between 0.45 and $0.90 \mathrm{mg} / \mathrm{g}$ inhibits hyaluronan synthesis. ${ }^{38} \mathrm{HLX}$ (50-300 units) is clinically used as a local adjuvant to increase the diffusion of local anesthetics. ${ }^{39}$ In our studies, HLX was subcutaneously administrated at a dose of 10 units. 4MU or HLX at the administration doses described in Figure 2A did not alter tumor volume (Supplementary Figure 1). Control mice were orally and subcutaneously administrated saline instead of 4MU/HLX. Control and 4MU/HLX-treated mice were then intravenously (i.v.) administrated ${ }^{89} \mathrm{Zr}$-labeled trastuzumab. Longitudinal PET imaging (Figure 2B) and biodistribution (Figure 2C, Supplementary Figure 2) of these mice at $48 \mathrm{~h}$ 
postadministration of ${ }^{89} \mathrm{Zr}$-labeled trastuzumab revealed a significant difference in the liver $(P<0.05$, Student's $t$-test), spleen $(P<0.05$, Student's $t$-test $)$, and tumor uptake $(P<0.001$, Student's $t$-test) of the radiotracer between the control vs 4MU/HLX tumors. JIMT1 control tumors had an uptake of $4.02 \pm 0.53 \%$ (mean \pm S.E.M.) of injected dose per gram (\% ID/g), while tumors treated with $4 \mathrm{MU} / \mathrm{HLX}$ yielded an uptake of $10.45 \pm 3.12 \%$ (mean \pm S.E.M.)

ID/g. The autoradiography analyses of control and 4MU/HLX-treated tumors further validated our findings from PET imaging and biodistribution (Supplementary Figure 3). A higher and homogeneous distribution of ${ }^{89} \mathrm{Zr}$-labeled trastuzumab radioactivity was observed in 4MU/HLX-treated mice when compared with control tumors. Additional biodistribution studies were performed in JIMT1 tumor-bearing mice that were blocked with a 40-fold excess of unlabeled trastuzumab. Antigen-blocked JIMT1 tumors showed similar uptake in control tumors and a significant reduction in ${ }^{89} \mathrm{Zr}$-labeled trastuzumab tumor uptake in 4MU/HLX-treated mice (Figure 2C). Additional PET imaging and biodistribution experiments demonstrated that the tumor uptake of a radiolabeled isotype control IgG was low and comparable in both control and 4MU/HLX-treated mice (Supplementary Figures 4 and 5).

Taken together, these studies support the potential of 4MU/HLX-mediated hyaluronan depletion to enhance the avidity of HER2-expressing JIMT1 tumors for trastuzumab.

\section{Depletion of Hyaluronan Decreases CD44, CAV1, and HER2-Signal Transduction in Jimt1 Breast Cancer Cells.}

Having found that hyaluronan depletion enhances trastuzumab accumulation in JIMT1 breast tumors (Figures 1 and 2), we next investigated how 4MU/HLX treatment would affect the JIMT1 cell sensitivity to trastuzumab therapy. JIMT1 cells were treated with 4MU, HLX, and 4MU/HLX in the presence and absence of trastuzumab. JIMT1 cell death, as determined by excluded trypan blue, was higher in 4MU/trastuzumab or 4MU/HLX/ trastuzumab when compared with respective control cells. Although trastuzumab, 4MU/ trastuzumab, and HLX/trastuzumab had some growth inhibitory effect in JIMT1 cells (Figure 3A), the addition of 4MU/HLX to trastuzumab significantly enhanced efficacy $(P<$ 0.05 , Student's $t$-test).

Given that CD44 is a transmembrane receptor for hyaluronan, ${ }^{17}$ we sought to determine if 4MU/HLX-mediated hyaluronan depletion downregulates CD44 protein expression. We observed that JIMT1 cells treated with 4MU, 4MU/ trastuzumab, and 4MU/HLX/ trastuzumab have lower CD44 protein levels when compared with control cells (Figure 3B,E). On the other hand, HLX treatment upregulated CD44 in JIMT1 cells (Figure 3B,E).

Our previous studies demonstrated a role for CAV1 protein on HER2 availability at the cell membrane for binding anti-HER2 antibodies, ${ }^{20,40}$ and recent studies demonstrated that JIMT1 cells do not exhibit a predominant localization for HER2 at the cell membrane. ${ }^{19}$ In this study, we performed immunoblot studies to detect changes in CAV1 upon 4MU/HLX/ trastuzumab treatment (Figure 3B,E). JIMT1 cells treated with 4MU/HLX or 4MU/HLX/ trastuzumab revealed a decrease in CAV1 protein when compared with control cells (Figure 3B,E). 
Next, we conducted immunoblot assays to investigate possible differences in the HER2signal transduction of JIMT1 control, 4MU, HLX, 4MU/HLX, trastuzumab, 4MU/ trastuzumab, HLX/trastuzumab, and 4MU/HLX/trastuzumab-treated cells (Figure 3B-D). Evaluation of HER-downstream intracellular signaling revealed that pHER2, pHER3, pEGFR, pERK, and pAKT were downregulated in 4MU/HLX/trastuzumab-treated cells compared with other treatment groups (Figure 3B,C). A decrease in protein phosphorylation occurred in the absence of total protein degradation as we did not observe changes in total HER2, HER3, EGFR, ERK, and AKT upon cell treatments. Knowing that HER2 dimerization induces autophosphorylation and further induction of downstream prooncogenic signaling pathways, ${ }^{3-5}$ we determined if changes in HER2-signal transduction are due to changes in HER2 dimerization. Immunoprecipitation assays demonstrated that HLX, trastuzumab, and 4MU/HLX/trastuzumab increase HER2-HER3 heterodimers in JIMT1 breast cancer cells (Figure 3B,D). Treatment of JIMT1 cells with HLX significantly enhanced phosphorylation of HER2, HER3, EGFR, and AKT, associated with an increase in HER2-HER3 dimers (Figure 3B-D). These results suggest that HLX treatment increases HER2-HER3 active dimers. An increase in HER2-HER3 dimers in JIMT1 cells treated with trastuzumab was also accompanied by a significant increase in pHER2, pHER3, and pAKT (Figure 3B,C). Although 4MU/HLX/trastuzumab treatment increased HER2-HER3 dimers (Figure 3B,D), we detected a reduction in phosphorylation of HER proteins, demonstrating that those are inactive dimers (Figure 3B-D).

Collectively, our results are consistent with a decrease in CD44, CAV1, and HER2-signal transduction upon treatment with 4MU/HLX/trastuzumab and the formation of non-activated HER2 dimerized receptors.

\section{Depletion of Hyaluronan Enhances Trastuzumab Therapeutic Efficacy in JIMT1 Breast Tumors.}

We showed that 4MU/HLX enhances trastuzumab accumulation in HER2-expressing JIMT1 tumors (Figures 1 and 2) and decreases in vitro HER2-downstream oncogenic signaling (Figure 3). To determine the in vivo efficacy of trastuzumab treatment when combined with 4MU/HLX, we conducted therapeutic studies in mice containing JIMT1 xenografts. Mice were orally administrated $4 \mathrm{MU}(0.8 \mathrm{mg} / \mathrm{g}$ of mice) every day for 5 weeks, subcutaneously injected with HLX (10 units) every day in the last 3 weeks, and treated weekly with $5 \mathrm{mg} / \mathrm{kg}$ trastuzumab (intraperitoneal administration) in the last 3 weeks (Figure 4A). Mice treated with either trastuzumab alone or trastuzumab combined with 4MU/HLX showed higher tumor growth inhibition when compared with the control, 4MU, HLX, 4MU/HLX, 4MU/ trastuzumab, and HLX/trastuzumab (Figure 4B-D). Additionally, the therapeutic efficacy of trastuzumab combined with 4MU/HLX was significantly improved compared with trastuzumab alone (Figure 4E, $P<0.001$, $t$-test). Therapy with 4MU/HLX/trastuzumab induced a significant prolongation of mice survival when compared with 4MU, HLX, 4MU/HLX (Supplementary Figure 6), 4MU/trastuzumab, HLX/trastuzumab, or trastuzumab alone (Figure 4F, $P<0.001$, Log-rank). 
Similar to our immunoblot results on CAV1 and CD44 proteins (Figure 3B,E), a decrease in CD44 and CAV1 protein levels was also observed in JIMT1 xenografts at 60 days posttherapy with 4MU/HLX or 4MU/HLX/trastuzumab (Figure 5).

These results demonstrate that 4MU/HLX-mediated hyaluronan depletion improves trastuzumab therapeutic efficacy in JIMT1 xenografts.

\section{DISCUSSION}

Trastuzumab and other monoclonal anti-HER2 antibodies (pertuzumab), HER2 tyrosine kinase inhibitors (lapatinib, neratinib, and pazopanib), and antibody-drug conjugates (TDM1) improve survival in patients with HER2-expressing breast cancer. ${ }^{2}$ Patients with HER2-overexpressing metastatic breast cancer show a clinical response of 35\% when treated with trastuzumab first-line therapy, ${ }^{41}$ and the combination of trastuzumab with taxanes or vinorelbine increases the response rate to $40-51 \% .{ }^{42}$ In the remaining patients, no tumor regression is observed, although $E R B B 2$ gene amplification and HER2 protein overexpression are present in primary tumor and metastases. Primary and acquired resistance mechanisms contribute to tumor resistance to HER2-targeted therapy. ${ }^{17}$ Our previous studies demonstrate that, in addition to HER2-positivity, HER2 membrane availability needs to be considered during patient selection for HER2-targeted therapies. ${ }^{20,40}$ In this context, the CD44/hyaluronan complex leads to diminished binding of trastuzumab to HER2-overexpressing breast tumors because this complex masks HER2 at the membrane for binding trastuzumab and mediates HER2 internalization. ${ }^{18}$

In this study, we extended our previous work on the pharmacologic modulation of HER2 membrane availability for binding trastuzumab and gained further insights on the potential of targeted degradation of the CD44/hyaluronan complex for improved antibody binding to HER $2^{+}$breast cancer cells. Previous studies have depleted hyaluronan synthesis using 4MU. 18 In our studies, HLX was used to deplete endogenously produced hyaluronan, and 4MU was used to inhibit hyaluronan synthesis. The HER2-overexpressing JIMT1 breast cancer cells were used in our studies as these cells are resistant to trastuzumab therapy, despite its ERBB2 amplification and HER2 protein overexpression. ${ }^{32}$ We found that pretreatment of JIMT1 breast cancer cells with HLX/ 4MU increases membrane-bound trastuzumab (Figure 1). We used HER2-targeted preclinical molecular imaging to show that HLX/4MU increases trastuzumab accumulation in JIMT1 xenografts (Figure 2). Therapeutic experiments demonstrated that the combination of trastuzumab with HLX/4MU decreases HER2mediated oncogenic signaling (Figure 3) and CD44 protein expression (Figures 3 and 5), improving trastuzumab efficacy (Figure 4).

HER2 availability at the cell membrane for binding trastuzumab is a complex biological process that is yet to be fully understood. While some studies suggest low HER2 internalization from the cell membrane to the intracellular compartment, ${ }^{43,44}$ others have demonstrated rapid recycling to the cell membrane upon endocytosis. ${ }^{45,46}$ These contradictory studies can be explained by recent findings demonstrating that HER2 trafficking and internalization is cell-line-dependent. ${ }^{19,20}$ In this context, JIMT1 cells exhibit an intracellular HER2 distribution as a result of receptor endocytosis. ${ }^{19}$ HER2 presence at 
the cell membrane is an important predictor of trastuzumab efficacy, as the antibody binds the extracellular domain of HER2. In JIMT1 breast cancer cells, the low availability of HER2 at the cellular membrane is associated with resistance to therapy using trastuzumab, ${ }^{34}$ pertuzumab, ${ }^{33}$ and trastuzumab-DM1 conjugate (TDM1). ${ }^{35,47}$ Previous studies have suggested a role for hyaluronan/CD44 in regulating HER2 endocytosis in JIMT1 cells. ${ }^{18}$ As a result, 4MU/HLX-mediated depletion of hyaluronan/CD44 (Figures 3 and 5) increases membrane-bound trastuzumab (Figures 1 and 4).

In previous work, we demonstrated that low availability of HER2 at the cell membrane is explained by endocytic trafficking systems mediating HER2 internalization. ${ }^{20}$ In vitro internalization assays demonstrated that, while HLX increases ${ }^{89} \mathrm{Zr}$-labeled trastuzumab internalization, 4MU/HLX enhances membrane-bound radioactivity (Figure 1). CAV1 is the main protein of the caveolae-endocytic pathway and mediates HER2 internalization from the cellular membrane to the intracellular compartment. ${ }^{20,47}$ Others have demonstrated that, at the cell membrane, CD44 exists in caveolae structures and binds CAV1. ${ }^{48}$ CAV1 and CD44 expression was detected in JIMT1 breast tumors (Figures 3 and 5). HLX-mediated trastuzumab internalization was associated with an increase in CD44 protein (Figures 1 and 3). The combination treatment 4MU/ HLX reduced CD44 and CAV1 protein levels both in vitro and in vivo (Figures 3 and 5), suggesting that the depletion of the CD44/hyaluronan interferes with the expression of endocytic proteins. In addition to the CD44/hyaluronan complex and endocytic proteins, the membrane-associated mucin 4 (MUC4) interferes with trastuzumab binding to the HER2 receptor in JIMT1 breast tumor cells. ${ }^{49}$ Further studies are necessary to determine changes in MUC4 protein levels after JIMT1 tumor treatment with 4MU/HLX.

Pretreatment of tumors with HLX increases intratumor drug concentrations, ${ }^{26}$ and the Herceptin Hylecta (i.e., a combination of trastuzumab with HLX) has been recently approved by the FDA. In addition to depletion of components of the extracellular matrix, HLX seems to affect antibody internalization as our results show a decrease in membranebound ${ }^{89} \mathrm{Zr}$-labeled trastuzumab accompanied by an increase in antibody internalization (Figure 1) in JIMT1 cells treated with HLX alone.

HER2 does not have a known ligand; however, the receptor occurs in a conformation (known as open position) that is naturally ready for binding other members of the HER family. ${ }^{3-5}$ Although HLX increased HER2-HER3 active dimers, the 4MU/HLX combination enhanced HER2-HER3 inactive dimers (Figure 3B). An increase in HER2 inactive dimers at the cell surface of tumor cells can explain the higher trastuzumab accumulation in 4MU/ HLX-treated tumors when compared with control tumors. The PI3K/AKT pathway and the RAS/Raf/MAPK signaling cascade are triggered by HER2 activation. ${ }^{17}$ Our in vitro studies demonstrated a decrease in HER2 downstream signaling upon the treatment of JIMT1 cells with 4MU/HLX/trastuzumab (Figure 3). Because our in vitro studies were performed in the absence of HER ligands, the superior activity of the 4MU/HLX/trastuzumab combination is most evident in vivo, where the ligands are present (Figure 4).

Although 4MU increased in vitro membrane-bound ${ }^{89} \mathrm{Zr}$-labeled trastuzumab, 4MU/ trastuzumab combination therapy demonstrated lower efficacy in vivo when compared with 
trastuzumab alone. Previous studies have reported that $4 \mathrm{MU}$ does not completely deplete hyaluronan in vivo. ${ }^{50}$ Although $4 \mathrm{MU}$ inhibits hyaluronan synthesis, $4 \mathrm{MU}$ is not able to deplete hyaluronan that was already present in the tumors at the time of study initiation. Therefore, HLX was used to perform targeted degradation of hyaluronan. HLX is also not able to completely deplete hyaluronan as it does not inhibit hyaluronan synthesis, and therefore, HLX/trastuzumab had lower therapeutic efficacy when compared with trastuzumab. In our in vivo studies, 4MU/HLX enhanced trastuzumab efficacy; this strategy effectively unmasked more HER2 sites in JIMT1 tumors by inhibiting hyaluronan and its synthesis with 4MU/HLX.

In our studies, we did not detect a decrease or loss in HER2 total protein levels following treatment with trastuzumab or 4MU/HLX/trastuzumab (Figures 3 and 5). Previous studies have demonstrated that breast cancer cells resistant to trastuzumab, which retained HER2 expression, remain sensitive to trastuzumab in vivo. ${ }^{51}$ This has been explained in part by trastuzumab-mediated ADCC, a process that requires HER2 available at the cell membrane for trastuzumab binding. Further studies in immunocompetent mice are necessary to determine if 4MU/HLX/trastuzumab therapy enhances antibody ADCC.

${ }^{89} \mathrm{Zr}$-Labeled trastuzumab PET imaging is a powerful noninvasive molecular imaging technique to monitor the affinity of the antibody to the HER2 target. ${ }^{16,20,29}$ Similar to our previous studies, ${ }^{16}$ preclinical molecular imaging shows a low accumulation of ${ }^{89} \mathrm{Zr}$-labeled trastuzumab in JIMT1 xenografts (Figure 2). Our approach using 4MU/HLX enhanced ${ }^{89} \mathrm{Zr}$ labeled trastuzumab tumor accumulation at $48 \mathrm{~h}$ after tracer injection, suggesting the use of this strategy to improve anti-HER2 antibody accumulation in breast tumors containing high levels of CD44/hyaluronan.

\section{CONCLUSION}

Our data on the role of the CD44/hyaluronan complex in trastuzumab therapy support the need to consider HER2 membrane availability, in addition to receptor positivity, during patient selection for trastuzumab therapy. In trastuzumab-resistant JIMT1 breast cancer cells, the CD44/hyaluronan complex reduces HER2 availability at the cell membrane for binding to trastuzumab as the complex masks the receptor-binding domain. 4MU/HLX-mediated depletion of the CD44/hyaluronan complex improves membrane-bound trastuzumab and therapeutic efficacy. Besides depleting CD44/hyaluronan, the 4MU/HLX treatment decreases endocytic proteins such as CAV1, which concomitantly arrests HER2 endocytosis. Further investigations are necessary to determine the relationship between CAV1 and CD44/ hyaluronan in breast cancer cells resistant to trastuzumab.

\section{Supplementary Material}

Refer to Web version on PubMed Central for supplementary material.

\section{ACKNOWLEDGMENTS}

The authors acknowledge the Radiochemistry and Molecular Imaging Probe Core and the Antitumor Assessment Core, which were supported by the NIH grant P30 CA08748. This study was supported in part by the Geoffrey Beene Cancer Research Center of MSKCC (J.S.L.), NIH NCI R35 CA232130 (J.S.L.), and R00 CA181492 
(N.T.V.). We gratefully acknowledge Mr. William H. and Mrs. Alice Goodwin and the Commonwealth Foundation for Cancer Research and The Center for Experimental Therapeutics of Memorial Sloan Kettering Cancer Center. P.M.R.P. acknowledges the Tow Foundation Postdoctoral Fellowship from the MSKCC Center for Molecular Imaging and Nanotechnology. P.M.R.P. acknowledges the Alan and Sandra Gerry Metastasis and Tumor Ecosystems Center of MSKCC. The figure shown in Table of Contents was made using Biorender.

\section{REFERENCES}

(1). Yarden Y; Pines G The ERBB network: at last, cancer therapy meets systems biology. Nat. Rev. Cancer 2012, 12, 553-563. [PubMed: 22785351]

(2). Hayes DF HER2 and breast cancer - a phenomenal success story. N. Engl. J. Med 2019, 381, 1284-1286. [PubMed: 31502769]

(3). Citri A; Yarden Y EGF-ERBB signalling: towards the systems level. Nat. Rev. Mol. Cell Biol 2006, 7, 505-516. [PubMed: 16829981]

(4). Tzahar E; PinkasKramarski R; Moyer JD; et al. Bivalence of EGF-like ligands drives the ErbB signaling network. EMBO J. 1997, 16, 4938-4950. [PubMed: 9305636]

(5). Zhang XW; Gureasko J; Shen K; Cole PA; Kuriyan J An allosteric mechanism for activation of the kinase domain of epidermal growth factor receptor. Cell 2006, 125, 1137-1149. [PubMed: 16777603]

(6). Moasser MM The oncogene HER2: its signaling and transforming functions and its role in human cancer pathogenesis. Oncogene 2007, 26, 6469-6487. [PubMed: 17471238]

(7). King CR; Kraus MH; Aaronson SA Amplification of a novel v-erbB-related gene in a human mammary-carcinoma. Science 1985, 229, 974-976. [PubMed: 2992089]

(8). Slamon DJ; Clark GM; Wong SG; Levin WJ; Ullrich A; Mcguire WL Human breast cancer: correlation of relapse and survival with amplification of the Her-2/neu oncogene. Science 1987 , 235, 177-182. [PubMed: 3798106]

(9). Hudziak RM; Lewis GD; Winget M; Fendly BM; Shepard HM; Ullrich A p185HER2 monoclonalantibody has antiproliferative effects in vitro and sensitizes human breast tumor cells to tumor necrosis factor. Mol. Cell. Biol 1989, 9, 1165-1172. [PubMed: 2566907]

(10). Carter P; Presta L; Gorman CM; et al. Humanization of an anti-p185HER2 antibody for human cancer therapy. Proc. Natl. Acad. Sci. U. S. A 1992, 89, 4285-4289. [PubMed: 1350088]

(11). Wolff AC; Hammond MEH; Allison KH; et al. Human epidermal growth factor receptor 2 testing in breast cancer: American Society of Clinical Oncology/College of American Pathologists Clinical Practice Guideline Focused Update. J. Clin. Oncol 2018, 36, 2105-2122. [PubMed: 29846122]

(12). Pereira PMR; Abma L; Henry KE; Lewis JS Imaging of human epidermal growth factor receptors for patient selection and response monitoring - From PET imaging and beyond. Cancer Lett. 2018, 419, 139-151. [PubMed: 29414302]

(13). Ulaner GA; Hyman DM; Ross DS; et al. Detection of HER2-positive metastases in patients with HER2-negative primary breast cancer using ${ }^{89} \mathrm{Zr}$-trastuzumab PET/CT. J. Nucl. Med 2016, 57 , 1523-1528. [PubMed: 27151988]

(14). Gebhart G; Lamberts LE; Wimana Z; et al. Molecular imaging as a tool to investigate heterogeneity of advanced HER2-positive breast cancer and to predict patient outcome under trastuzumab emtansine (T-DM1): the ZEPHIR trial. Ann. Oncol 2016, 27, 619-624. [PubMed: 26598545]

(15). Sanchez-Vega F; Hechtman JF; Castel P; et al. EGFR and MET amplifications determine response to HER2 inhibition in ERBB2-amplified esophagogastric cancer. Cancer Discovery 2019, 9, 199-209. [PubMed: 30463996]

(16). McKnight BN; Viola-Villegas NT Monitoring Src status after dasatinib treatment in HER2+ breast cancer with ${ }^{89}$ Zr-trastuzumab PET imaging. Breast Cancer Res. 2018, 20, 130-142. [PubMed: 30359299]

(17). Pohlmann PR; Mayer IA; Mernaugh R Resistance to trastuzumab in breast cancer. Clin. Cancer Res 2009, 15, 7479-7491. [PubMed: 20008848] 
(18). Palyi-Krekk Z; Barok M; Isola J; Tammi M; Szöllosi J; Nagy P Hyaluronan-induced masking of ErbB2 and CD44-enhanced trastuzumab internalisation in trastuzumab resistant breast cancer. Eur. J. Cancer 2007, 43, 2423-2433. [PubMed: 17911008]

(19). Pietila M; Sahgal P; Peuhu E; Jantti NZ; Paatero I; Narva E; Al-Akhrass H; Lilja J; Georgiadou M; Andersen OM; Padzik A; Sihto H; Joensuu H; Blomqvist M; Saarinen I; Bostrom PJ; Taimen P; Ivaska J SORLA regulates endosomal trafficking and oncogenic fitness of HER2. Nat. Commun 2019, 10, 2340. [PubMed: 31138794]

(20). Pereira PMR; Sharma SK; Carter LM; Edwards KJ; Pourat J; Ragupathi A; Janjigian YY; Durack JC; Lewis JS Caveolin-1 mediates cellular distribution of HER2 and affects trastuzumab binding and therapeutic efficacy. Nat. Commun 2018, 9, 9. [PubMed: 29339724]

(21). Yang L; Li Y; Bhattacharya A; Zhang YS A recombinant human protein targeting HER2 overcomes drug resistance in HER2-positive breast cancer. Sci. Transl. Med 2019, 11, 11.

(22). Varadi T; Mersich T; Auvinen P; et al. Binding of trastuzumab to ErbB2 is inhibited by a high pericellular density of hyaluronan. J. Histochem. Cytochem 2012, 60, 567-575. [PubMed: 22562558]

(23). Naor D; Sionov RV; IshShalom D CD44: structure, function, and association with the malignant process. Adv. Cancer Res 1997, 71, 241-319. [PubMed: 9111868]

(24). Bourguignon LYW; Zhu HB; Zhou B; Diedrich F; Singleton PA; Hung MC Hyaluronan promotes CD44v3-Vav2 interaction with Grb2-p185(HER2) and induces Rac1 and Ras signaling during ovarian tumor cell migration and growth. J. Biol. Chem 2001, 276, 48679-48692. [PubMed: 11606575]

(25). Bookbinder LH; Hofer A; Haller MF; et al. A recombinant human enzyme for enhanced interstitial transport of therapeutics. J. Controlled Release 2006, 114, 230-241.

(26). Whatcott CJ; Han H; Posner RG; Hostetter G; Von Hoff DD Targeting the tumor microenvironment in cancer: why hyaluronidase deserves a second look. Cancer Discovery 2011, 1, 291-296. [PubMed: 22053288]

(27). Shpilberg O; Jackisch C Subcutaneous administration of rituximab (MabThera) and trastuzumab (Herceptin) using hyaluronidase. Br. J. Cancer 2013, 109, 1556-1561. [PubMed: 24002601]

(28). Xu KM; Lee F; Gao SJ; Tan MH; Kurisawa M Hyaluronidase-incorporated hyaluronic acidtyramine hydrogels for the sustained release of trastuzumab. J. Controlled Release 2015, 216, 4755.

(29). Janjigian YY; Viola-Villegas N; Holland JP; et al. Monitoring afatinib treatment in HER2positive gastric cancer with ${ }^{18}$ F-FDG and ${ }^{89} \mathrm{Zr}$-trastuzumab PET. J. Nucl. Med 2013, 54, $936-$ 943. [PubMed: 23578997]

(30). Holland JP; Sheh YC; Lewis JS Standardized methods for the production of high specific-activity zirconium-89. Nucl. Med. Biol 2009, 36, 729-739. [PubMed: 19720285]

(31). Escorcia FE; Steckler JM; Abdel-Atti D; et al. Tumor-specific Zr-89 immuno-PET imaging in a human bladder cancer model. Mol. Imaging Biol 2018, 20, 808-815. [PubMed: 29508263]

(32). Tanner M; Kapanen AI; Junttila T; et al. Characterization of a novel cell line established from a patient with Herceptin-resistant breast cancer. Mol. Cancer Ther 2004, 3, 1585-1592. [PubMed: 15634652]

(33). Toth G; Szoor A; Simon L; Yarden Y; Szollosi J; Vereb G The combination of trastuzumab and pertuzumab administered at approved doses may delay development of trastuzumab resistance by additively enhancing antibody-dependent cell-mediated cytotoxicity. Mabs-Austin. 2016, 8, 1361-1370.

(34). O'Brien NA; Browne BC; Chow L; et al. Activated phosphoinositide 3-kinase/AKT signaling confers resistance to trastuzumab but not lapatinib. Mol. Cancer Ther 2010, 9, 1489-1502. [PubMed: 20501798]

(35). Li JY; Perry SR; Muniz-Medina V; et al. A biparatopic HER2-targeting antibody-drug conjugate induces tumor regression in primary models refractory to or ineligible for HER2-targeted therapy. Cancer Cell 2019, 35, 948-949. [PubMed: 31185215]

(36). Pályi-Krekk Z; Barok M; Kovács T; et al. EGFR and ErbB2 are functionally coupled to CD44 and regulate shedding, internalization and motogenic effect of CD44. Cancer Lett. 2008, 263, 231-242. [PubMed: 18276068] 
(37). Lokeshwar VB; Mirza S; Jordan A Targeting hyaluronic acid family for cancer chemoprevention and therapy. Adv. Cancer Res 2014, 123, 35-65. [PubMed: 25081525]

(38). Lokeshwar VB; Lopez LE; Munoz D; et al. Antitumor activity of hyaluronic acid synthesis inhibitor 4-methylumbelliferone in prostate cancer cells. Cancer Res. 2010, 70, 2613-2623. [PubMed: 20332231]

(39). Buhren BA; Schrumpf H; Hoff NP; Bölke E; Hilton S; Gerber PA Hyaluronidase: from clinical applications to molecular and cellular mechanisms. Eur. J. Med. Res 2016, 21, 21. [PubMed: 27165726]

(40). Pereira PMR; Mandleywala K; Ragupathi A; Carter LM; Goos JACM; Janjigian YY; Lewis JS Temporal modulation of HER2 membrane availability increases pertuzumab uptake and pretargeted molecular imaging of gastric tumors. J. Nucl. Med 2019, 60, 1569. [PubMed: 31171598]

(41). Vogel CL; Cobleigh MA; Tripathy D; Gutheil JC; Harris LN; Fehrenbacher L; Slamon DJ; Murphy M; Novotny WF; Burchmore M; Shak S; Stewart SJ First-line Herceptin monotherapy in metastatic breast cancer. Oncology 2001, 61, 37-42. [PubMed: 11694786]

(42). Burstein HJ; Keshaviah A; Baron AD; et al. Trastuzumab plus vinorelbine or taxane chemotherapy for HER2-overexpressing metastatic breast cancer: the trastuzumab and vinorelbine or taxane study. Cancer 2007, 110, 965-972. [PubMed: 17614302]

(43). Kraus MH All ErbB receptors other than the epidermal growth factor receptor are endocytosis impaired. J. Biol. Chem 1996, 271, 5251-5257. [PubMed: 8617810]

(44). Hommelgaard AM; Lerdrup M; van Deurs B Association with membrane protrusions makes ErbB2 an internalization-resistant receptor. Mol. Biol. Cell 2004, 15, 1557-1567. [PubMed: 14742716]

(45). Harari D; Yarden Y Molecular mechanisms underlying ErbB2/HER2 action in breast cancer. Oncogene 2000, 19, 6102-6114. [PubMed: 11156523]

(46). Hendriks BS; Opresko LK; Wiley HS; Lauffenburger D Coregulation of epidermal growth factor receptor/human epidermal growth factor receptor 2 (HER2) levels and locations: Quantitative analysis of HER2 overexpression effects. Cancer Res. 2003, 63, 1130-1137. [PubMed: 12615732]

(47). Sung M; Tan XZ; Lu BW; et al. Caveolae-mediated endocytosis as a novel mechanism of resistance to trastuzumab emtansine (T-DM1). Mol. Cancer Ther 2018, 17, 243-253. [PubMed: 29054985]

(48). Lee JL; Wang MJ; Sudhir PR; Chen JY CD44 engagement promotes matrix-derived survival through the CD44-Srcintegrin axis in lipid rafts. Mol. Cell. Biol 2008, 28, 5710-5723. [PubMed: 18644869]

(49). Nagy P; Friedlander E; Tanner M; et al. Decreased accessibility and lack of activation of ErbB2 in JIMT-1, a herceptin-resistant, MUC4-Expressing breast cancer cell line. Cancer Res. 2005, 65, 473-482. [PubMed: 15695389]

(50). Nagase H; Kudo D; Suto A; et al. 4-Methylumbelliferone suppresses hyaluronan synthesis and tumor progression in SCID mice intra-abdominally inoculated with pancreatic cancer cells. Pancreas 2017, 46, 190-197. [PubMed: 27846148]

(51). Kute TE; Savage L; Stehle JR; Kim-Shapiro JW; Blanks MJ; Wood J; Vaughn JP; et al. Breast tumor cells isolated from in vitro resistance to trastuzumab remain sensitive to trastuzumab antitumor effects in vivo and to ADCC killing. Cancer Immunol. Immunother 2009, 58, 1887-1896. [PubMed: 19340424] 

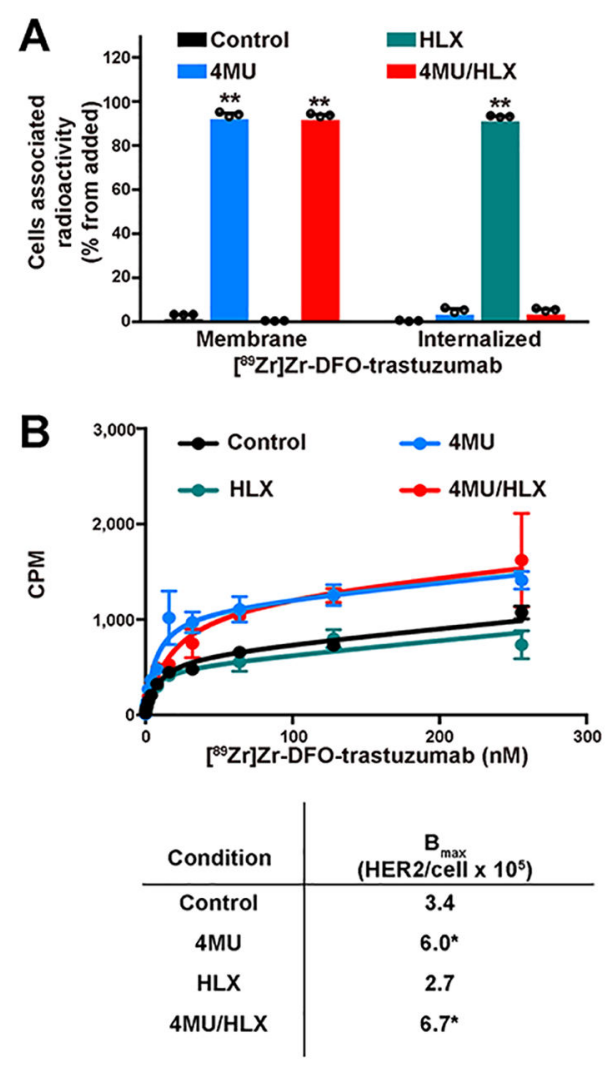

Figure 1.

Treatment with 4MU/HLX increases [ $\left.{ }^{89} \mathrm{Zr}\right] \mathrm{Zr}$-DFO-trastuzumab binding to HER2expressing JIMT1 breast cancer cells.(A) Membrane-bound and internalized [ ${ }^{89} \mathrm{Zr}$ Zr-DFOtrastuzumab in JIMT1 breast cancer cells before and after treatment with 4MU, HLX, or 4MU/HLX (bars, $n=3$, mean \pm S.E.M, ${ }^{*} * P<0.01$ based on a Student's $t$-test and compared with control). Cells were treated with $1 \mathrm{mM} 4 \mathrm{MU}$ for $48 \mathrm{~h}, 1$ Unit HLX for $2 \mathrm{~h}$, or 4MU/HLX in the presence of $1 \mu \mathrm{M}$ [ $\left.{ }^{89} \mathrm{Zr}\right] \mathrm{Zr}$-DFO-trastuzumab for $1.5 \mathrm{~h}$. (B) Binding kinetics of [ $\left.{ }^{89} \mathrm{Zr}\right] \mathrm{Zr}$-DFO-trastuzumab in control, 4MU, HLX, or 4MU/HLX-treated JIMT1 cells. JIMT1 control and treated cells were incubated with $\left[{ }^{89} \mathrm{Zr}\right] \mathrm{Zr}-\mathrm{DFO}$-trastuzumab $(0-256 \mathrm{nM})$ for $3 \mathrm{~h}$ at $4{ }^{\circ} \mathrm{C}$ (upper panel). Data are presented as the mean \pm S.E.M, $n=3$. Binding parameters (lower panel) of ${ }^{89} \mathrm{Zr}$-labeled trastuzumab to JIMT1 control and treated cells ( ${ }^{*} P<0.05$ based on a Student's $t$-test and compared with control). 

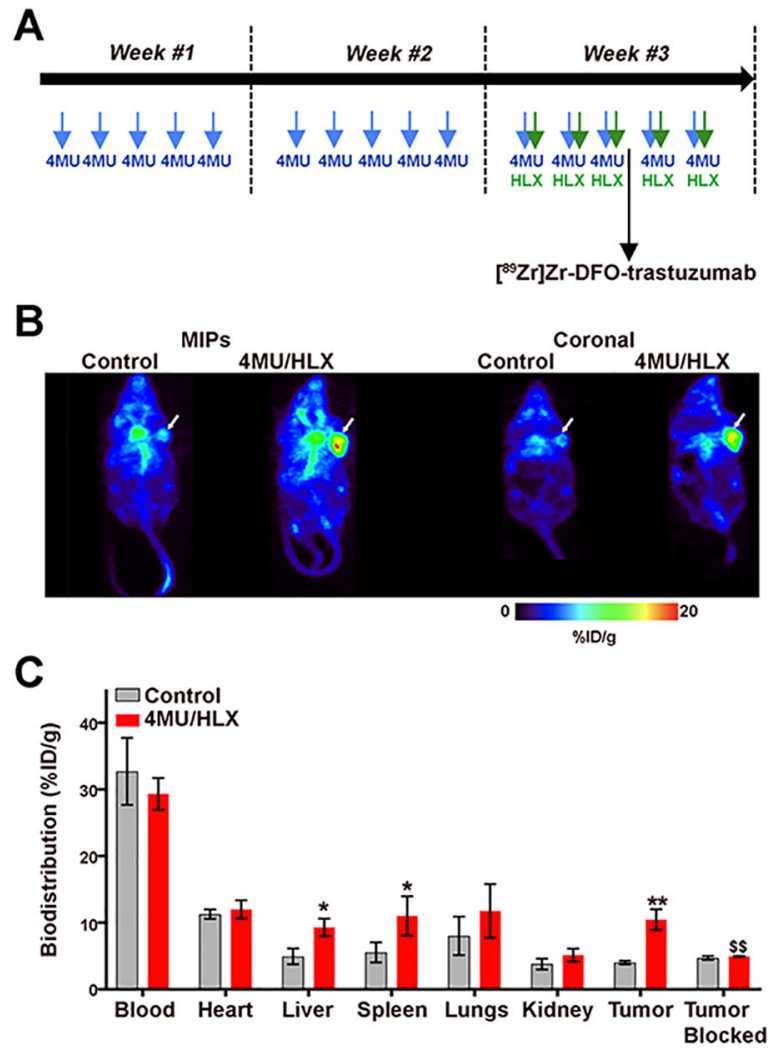

Figure 2.

Treatment with 4MU/HLX increases [ $\left.{ }^{89} \mathrm{Zr}\right] \mathrm{Zr}$-DFO-trastuzumab accumulation in HER2expressing JIMT1 tumor xenografts, an effect that is blocked with an excess of unlabeled trastuzumab. (A) Schematic diagram of JIMT1 tumor-bearing mice treated with 4MU/HLX and used in imaging and biodistribution studies with $\left[{ }^{89} \mathrm{Zr}\right] \mathrm{Zr}$-DFO-trastuzumab. 4MU was orally administered ( $0.8 \mathrm{mg} / \mathrm{g}$ of mice) every day for 3 weeks. HLX was subcutaneously administered (10 units) every day in the third week. [ ${ }^{89} \mathrm{Zr}$ ]Zr-DFO-trastuzumab (8.14-10.18 $\mathrm{MBq}, 80-100 \mu \mathrm{g}$ of protein) was administered by tail vein injection on day 3 of the third week. (B) Representative MIP and coronal PET images and (C) biodistribution at $48 \mathrm{~h}$ p.i. of $\left[{ }^{89} \mathrm{Zr}\right] \mathrm{Zr}$-DFO-trastuzumab in control and 4MU/HLX-treated athymic nude mice bearing subcutaneous JIMT1 breast tumors. Blocking experiments were performed by the administration of ${ }^{89} \mathrm{Zr}$-labeled trastuzumab in the presence of a 40 -fold molar excess of unlabeled trastuzumab. Data represent the mean $\pm \operatorname{SEM}(n=5$ mice per group, $* P<0.05$, $* * P<0.01$ based on a Student's $t$-test and compared with the control; ${ }^{\$} \$ P<0.01$ based on a Student's $t$-test and compared with tumor treated with 4MU/HLX). Treatments were performed as represented in panel A. White arrows show the tumor region. MIPs, maximum intensity projection. $\% \mathrm{ID} / \mathrm{g}$, percentage of injected dose per gram of tissue. 


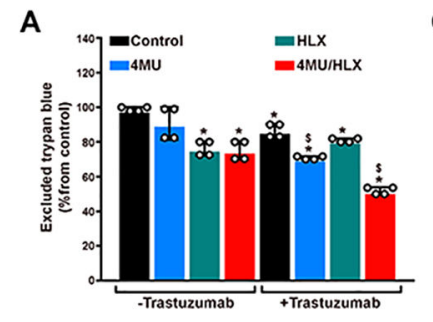

B

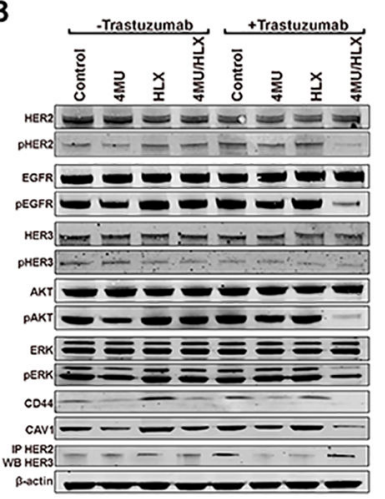

C
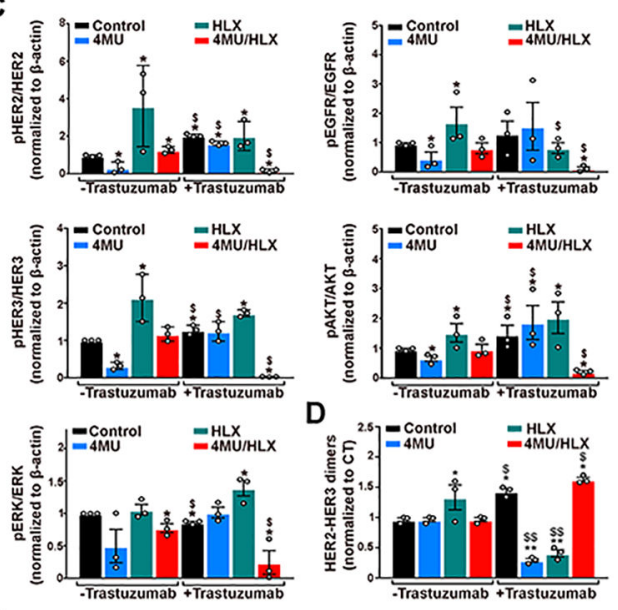

$E$
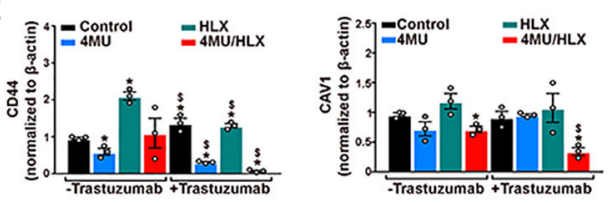

Figure 3.

Treatment with 4MU/HLX increases in vitro trastuzumab efficacy and decreases HER2signal transduction in JIMT1 breast cancer cells. (A) Excluded trypan blue, (B) protein expression and activation of HER family receptors pathways, CD44, and CAV1 proteins as analyzed and (C,D,E) quantified by Western blot in JIMT1 breast cancer cells in the presence or absence of trastuzumab and after treatment with 4MU, HLX, or 4MU/HLX. Cells were treated with $1 \mathrm{mM} 4 \mathrm{MU}$ for $48 \mathrm{~h}, 1$ Unit HLX for $2 \mathrm{~h}$, or 4MU/HLX in the presence of $20 \mathrm{nM}$ trastuzumab for 24 h. (A) Bars, $n=4$, mean \pm S.E.M, $* * P<0.01$ based on a Student's $t$-test, * compared with control group, $\$$ compared with the nontrastuzumab respective group. (C, D, E) Bars, $n=3$, mean \pm S.E.M, $* P<0.05$ based on a Student's $t$-test and compared with the control group, ${ }^{\$} P<0.05$ based on a Student's $t$-test and compared with the nontrastuzumab respective group. 
A

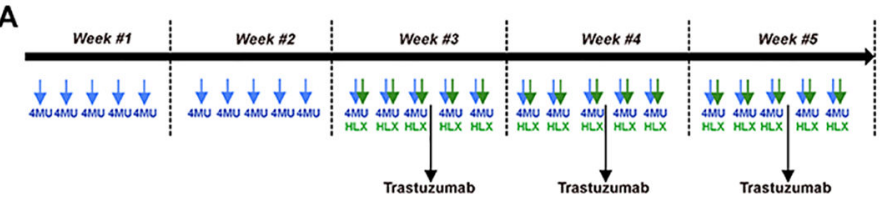

B
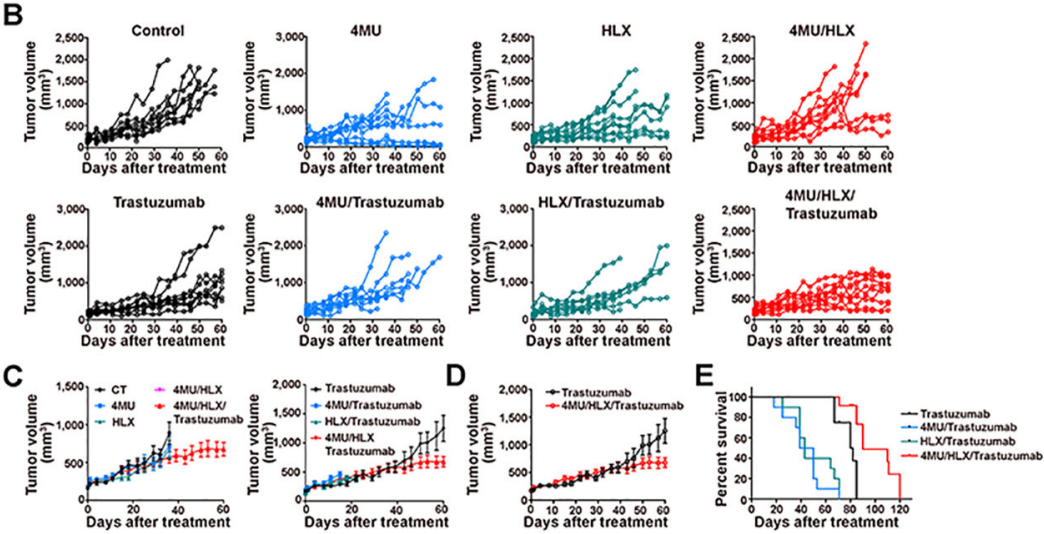

Figure 4.

4MU/HLX treatment increases trastuzumab efficacy in JIMT1 tumor xenografts. (A)

Schematic diagram of JIMT1 tumor-bearing mice treated with 4MU, HLX, or 4MU/HLX in the presence and absence of trastuzumab. 4MU was orally administrated $(0.8 \mathrm{mg} / \mathrm{g}$ of mice $)$ every day for 5 weeks. 4MU administration started on day 0. HLX was subcutaneously administered (10 units) every day in the third, fourth, and fifth weeks. Intraperitoneal trastuzumab administration $5 \mathrm{mg} / \mathrm{kg}$ weekly (during 3 weeks) was started on the third week. (B, C, D) Tumor volumes $\left(\mathrm{mm}^{3}\right)$ as a function of time in control, 4MU, HLX, 4MU/HLX, trastuzumab, trastuzumab/4MU, trastuzumab/HLX, and trastuzumab/4MU/HLX in $n u / n u$ female mice bearing JIMT1 breast tumors ( $n=10$ mice per group). (E) Kaplan-Meier survival curves of mice bearing JIMT1 breast tumors and treated with trastuzumab, 4MU/ trastuzumab, HLX/trastuzumab, or 4MU/HLX/trastuzumab. 

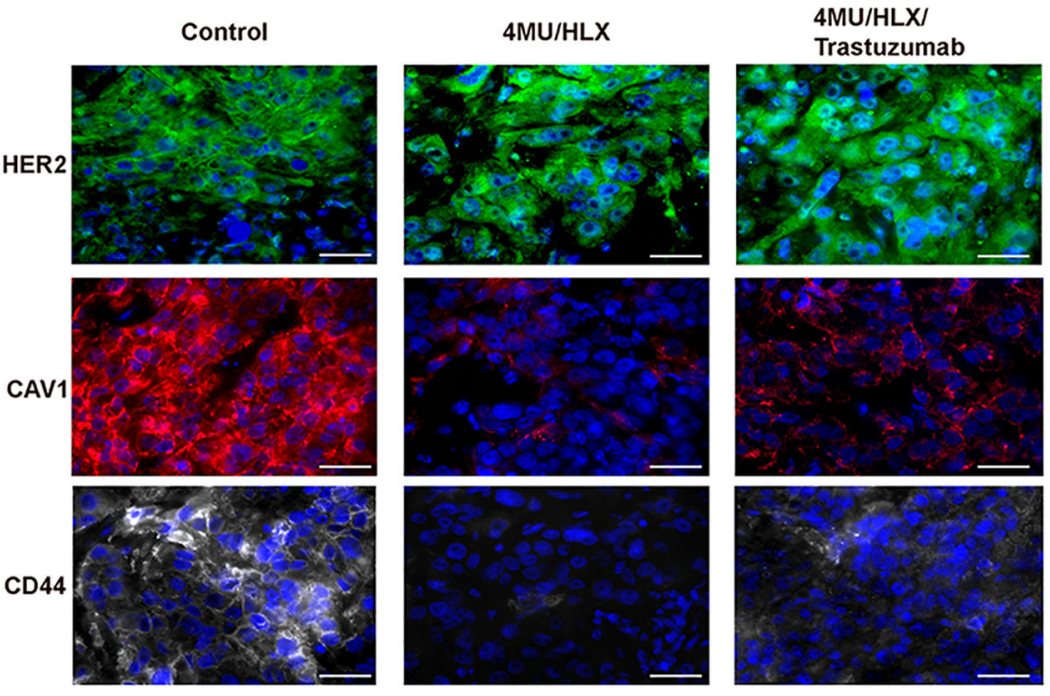

Figure 5.

4MU/HLX treatment decreases CD44 and CAV1 protein expression in JIMT1 tumor xenografts. HER2, CAV1, and CD44 immunofluorescence in control, 4MU/HLX, and trastuzumab/4MU/HLX JIMT1 breast tumors. JIMT1 tumor-bearing mice were treated with 4MU/HLX or 4MU/HLX/trastuzumab, as demonstrated in Figure 4A, and tumors were collected at 60 days post-therapy. Tumors were stained for HER2 (green), CAV1 (red), CD44 (gray), and DAPI (blue), and images were acquired on a fluorescence microscope. Scale bars, $50 \mu \mathrm{m}$. 